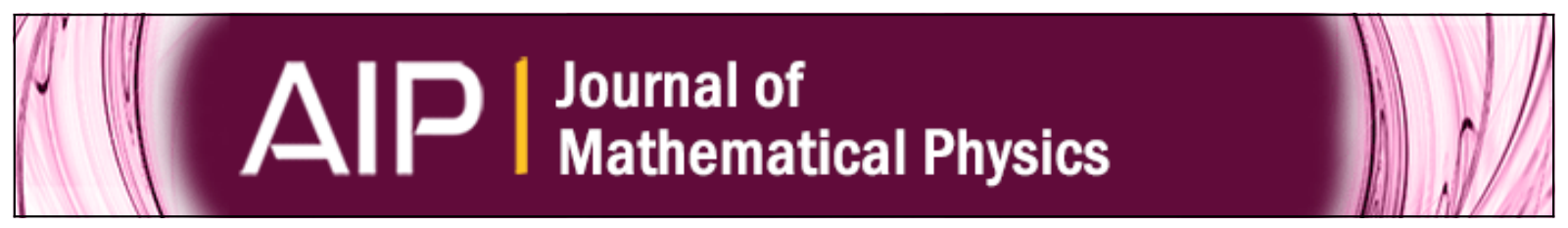

\title{
Electronic states on a lattice in periodic magnetic fields
}

Francisco Guil

Citation: Journal of Mathematical Physics 48, 033503 (2007); doi: 10.1063/1.2710199

View online: http://dx.doi.org/10.1063/1.2710199

View Table of Contents: http://scitation.aip.org/content/aip/journal/jmp/48/3?ver=pdfcov

Published by the AIP Publishing

Articles you may be interested in

Undulator radiation in a periodic magnetic field with a constant component

J. Appl. Phys. 104, 124507 (2008); 10.1063/1.3039094

Exact solution of the problem of an electron in a magnetic field consisting of a uniform field and arbitrarily arranged magnetic strings parallel to the uniform field

Low Temp. Phys. 28, 845 (2002); 10.1063/1.1528576

Erratum: "Field pattern of a magnetic dipole" [Am. J. Phys. 68, 577-578 (2000)]

Am. J. Phys. 69, 1112 (2001); 10.1119/1.1362697

Field pattern of a magnetic dipole

Am. J. Phys. 68, 577 (2000); 10.1119/1.19487

Effect of magnetic flux trapped in intergranular space on magnetic field dependences of $\mathrm{rf}$ absorption in HTS materials

Low Temp. Phys. 23, 122 (1997); 10.1063/1.593343

\section{Did your publisher get}

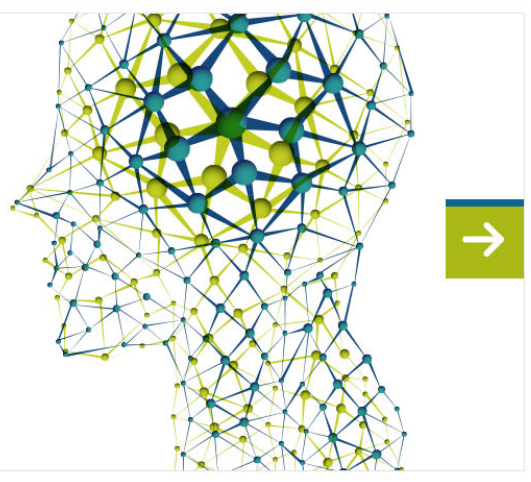




\title{
Electronic states on a lattice in periodic magnetic fields
}

\author{
Francisco Guil ${ }^{\text {a) }}$ \\ Departamento de Física Teórica, Facultad de Ciencias Físicas, Universidad Complutense, \\ 28040 Madrid, Spain
}

(Received 12 December 2006; accepted 12 January 2007; published online 5 March 2007)

The Hamiltonian for an electron in $\mathbb{Z}^{2}$, interacting with a perpendicular periodic magnetic field, is shown to be gauge equivalent to a periodic Hamiltonian. The wave function of the system is computed in terms of a ground state of the continuous motion in $\mathbb{R}^{2}$ for the Pauli Hamiltonian. Explicit expressions for the states, in both the Coulomb and Landau gauges, are considered. (0) 2007 American Institute of Physics. [DOI: 10.1063/1.2710199]

\section{INTRODUCTION}

Since the appearance of Hofstadter's paper, ${ }^{1}$ the study of electronic spectra in a lattice under the influence of a perpendicular magnetic field has become a field of continuous interest. For both uniform and periodic magnetic fields, a common feature of all studies in this subject consists in the numerical analysis of the energy levels. The resulting spectral structure leads to a scheme of energy bands, for a fixed rational value of the magnetic flux on an elementary cell of the lattice. ${ }^{2}$ Alternatively, the dependence of the energy on that rational flux ${ }^{3,4}$ produces a fractal figure, the Hofstadter's butterfly. For a uniform magnetic field, the problem is reduced to a one-dimensional problem, the Harper's equation that can be solved in the context of the theory of quantum group representations. ${ }^{5}$ The case of modulated fields in the tight-binding model is so far less generically treated. The usual approach to this problem consists in the construction, for concrete, simple enough magnetic fields, of Schrödinger equations with periodic coefficients. According to Bloch's theorem, states are given by periodic functions modulated by plane waves on the lattice.

The continuous motion of an electron in $\mathbb{R}^{2}$ under the influence of a perpendicular periodic magnetic field is described by the Pauli operator. It was considered, in the form we are interested in, by Dubrovin and Novikov in Refs. 6 and 7, where they gave a beautiful description of the ground states of the electron in terms of a vector bundle over the torus of quasimomenta based on the Weierstrass elliptic function theory. An alternative approach to this problem is given in Ref. 8 . Recently, the fractal nature of the spectra ${ }^{9,10}$ has become an object of interest. As applications, one can cite two physical problems whose understanding depends on the previous formulations: the quantum Hall effect ${ }^{11}$ and the flux-phase conjecture ${ }^{12-14}$. For many other physical implications and related questions in connection with the theory of electrons in magnetic fields, we submit to the reader to the literature cited.

In this paper we solve the Schrödinger equation for the Hamiltonian of an electron on a plane lattice with a periodic magnetic field. The periodic magnetic field, perpendicular to the plane lattice, is arbitrary except for the restriction on the values of the flux through its periodic parallelogram. For the case considered in the present paper, the flux is an integer multiple of $2 \pi$. This restriction gives two commuting magnetic translation operators. ${ }^{6}$ The main result of this paper is to point out the relation between these symmetries and a gauge transformation which makes the Hamiltonian translation invariant. Thus, despite the lack of translation invariance the coupling

${ }^{a)}$ Electronic mail: fguil@fis.ucm.es 
with the periodic magnetic field does not remove the degeneracy of the energy bands. This fact was already observed for the ground state of the continuous motion, ${ }^{6}$ although in this case there is no gauge equivalence with a periodic system.

The Hamiltonian

$$
H=\sum_{\mathbb{Z}^{2}} t_{a b} c_{a}^{+} c_{b}
$$

describes a system of free electrons in the plane lattice $\mathbb{Z}^{2}{ }^{12}$ The creation $c_{a}^{+}$and annihilation $c_{a}$ operators for spinless fermions satisfy the relations $c_{a} c_{b}+c_{b} c_{a}=0, c_{a}^{+} c_{b}+c_{b} c_{a}^{+}=\delta_{a b}$, and the coefficients $t_{a b}$ determine the hopping amplitudes for sites $a$ and $b$. It is assumed that $t_{a b}=\bar{t}_{b a}$ and $t_{a b}$ $=0$ if $a$ and $b$ are not connected by a bond. When $t_{a b}$ is a complex number, its argument represents the interaction of the electrons with a magnetic field perpendicular to the plane containing the electrons. In terms of the potential vector $A$ it can be written as

$$
t_{a b}=\left|t_{a b}\right| \exp -i \int_{a}^{b} A,
$$

where the integral is defined as the line integral of $A$ along the bond connecting sites $a$ and $b$. States for one electron, in the Hilbert space $\ell^{2}\left(Z^{2}\right)$, are described in the orthonormal basis $c_{a}^{+}|0\rangle$ by a vector

$$
\psi=\sum_{\mathbb{Z}^{2}} \psi(a) c_{a}^{+}|0\rangle
$$

where the coefficients $\psi(a)$ are the values of the wave function on $\mathbb{Z}^{2}$. For a system of electrons the state is the antisymmetric product of the individual states. The Schrödinger equation for the wave function of an electron,

$$
H \psi=E \psi,
$$

is just the spectral problem for the matrix $t_{a b}$,

$$
\sum_{b \in Z^{2}} t_{a b} \psi(b)=E \psi(a) .
$$

From now on, we shall limit ourselves to the case of a square lattice where $\left|t_{a b}\right|=1$ if $a$ and $b$ are nearest neighbors and zero in other case. In this situation, Eq. (1.2) reads

$$
u_{1}(x, y) \psi(x-1, y)+u_{1}^{-1}(x+1, y) \psi(x+1, y)+u_{2}(x, y) \psi(x, y-1)+u_{2}^{-1}(x, y+1) \psi(x, y+1)=E \psi(x, y),
$$

at each point $a=(x, y)$ in $\mathbb{Z}^{2}$. The functions $u_{1}(x, y)$ and $u_{2}(x, y)$ are the nonzero hopping amplitudes in the matrix $t$, which are given by the formulas

$$
u_{1}(x, y)=\exp i \int_{x-1}^{x} A_{1}\left(x^{\prime}, y\right) \mathrm{d} x^{\prime}, \quad u_{2}(x, y)=\exp i \int_{y-1}^{y} A_{2}\left(x, y^{\prime}\right) \mathrm{d} y^{\prime}
$$

for the vector components of $A=\left(A_{1}, A_{2}\right)$. The representation [Eq. (1.3)] of Eq. (1.2) in terms of the amplitudes $\psi(x, y)$ of $\psi$ allows us to consider solutions $\psi(x, y)$ which are not in $\ell^{2}\left(\mathbb{Z}^{2}\right)$.

The organization and content of the paper are as follows. In the next section we shall describe the gauge transformation that reduces Eq. (1.3) for an electron in a doubly periodic magnetic field, with a flux which is an integer multiple of $2 \pi$, to a periodic equation. The quantization of the flux increases the symmetry of the problem with two compatible magnetic translations [Eq. (2.3)]. The construction of the gauge transformation depends upon such symmetry. In Sec. III this transformation will be explicitly obtained for the Coulomb gauge which gives a connection with the 
continuous problem. As a result of this formulation, states in the tight-binding model for the electronic motion in the lattice correspond with ground states for the continuous motion. Although of theoretical interest, the Coulomb gauge is not suitable for explicit computations. We shall describe, in Sec. IV, the form of the equations in the Landau gauge. In this gauge, our formulas are given in terms of the Jacobi theta function in contrast to the Coulomb gauge where use is made of the Weierstrass theory for elliptic functions. Formulas (4.3) allow us to compute the energy spectrum in simple cases. The pictures exhibit the characteristic properties pointed out, for instance, in Ref. 2.

\section{GAUGE INVARIANCE}

If we assume that the Hamiltonian [Eq. (1.1)] is gauge invariant, we can give an interpretation of the complex amplitudes $t_{a b}$ in terms of the interaction of an electron with a magnetic field $B$. Let us consider a magnetic field $B$, perpendicular to the plane of the lattice, that can be written in terms of the vector potential $A$ according to the formula $B=\partial A_{2} / \partial x-\partial A_{1} / \partial y$. For the equivalent vector $A^{\prime}=A+\nabla \theta$, the new hopping amplitudes and wave function are

$$
\begin{gathered}
u_{k}^{\prime}(a)=u_{k}(a) \exp i\left(\theta(a)-\theta\left(a-e_{k}\right)\right), \quad k=1,2, \\
\psi^{\prime}(a)=\psi(a) \exp i \theta(a),
\end{gathered}
$$

as imposed by the invariance of Eq. (1.3). In the orthonormal basis $\left\{e_{1}, e_{2}\right\}$ points in the lattice, with coordinates $(x, y)$, are written in the form $a=x e_{1}+y e_{2}$. Thus we see that the amplitudes $\psi(a)$ and $\psi^{\prime}(a)$ are equivalent. The physical meaning of the magnetic field $B$ manifests in the following fact. Let us write the gauge invariant relation between $B$ and $A$ in integral form. We denote by $\Phi(a)$ the flux of $B$ through the elementary cell in the lattice having the point $a$ as its upper right corner. Then, according to the Stokes formula, we get the relation

$$
e^{i \Phi(a)}=\frac{u_{1}\left(a-e_{2}\right)}{u_{1}(a)} \frac{u_{2}(a)}{u_{2}\left(a-e_{1}\right)},
$$

which is gauge independent. In particular, the choice $A_{2}=0$ corresponds to the Landau gauge and reduces this formula to the relation

$$
u_{1}\left(a-e_{2}\right)=u_{1}(a) e^{i \Phi(a)} .
$$

It then follows that the coefficients $u_{1}$ in Eq. (1.3) and hence the energy $E$ are determined by the elementary fluxes $\Phi(a) .{ }^{12}$ In this gauge the magnetic field $B$ is equal to $-\partial A_{1} / \partial y$, thus we can take $u_{1}(x, 0)=1$ by setting $A_{1}(x, 0)=0$.

The states $\psi$ which are solutions of Eq. (1.3) as well as the energy spectrum of the Hamiltonian $H$ can be described, for the class of doubly periodic magnetic fields,

$$
B\left(x+l_{1}, y\right)=B\left(x, y+l_{2}\right)=B(x, y),
$$

by means of the elliptic function theory. Periods $l_{1}$ and $l_{2}$ of the field are assumed to be integer numbers, in order for $B(x, y)$ to be a periodic function on the lattice. Most of our formulas otherwise would not make sense. Although coefficients $u_{1}$ and $u_{2}$ for an arbitrary gauge are not periodic, we shall show that there exists a gauge where they are. To see that, we observe that, under a translation in the field periods, the vector potential $A$ undergoes variations given by the equations

$$
\begin{aligned}
& A\left(x-l_{1}, y\right)=A(x, y)+\nabla \phi_{1}, \\
& A\left(x, y-l_{2}\right)=A(x, y)+\nabla \phi_{2} .
\end{aligned}
$$

Assume that the values of the flux of $B$ are 


$$
\int_{0}^{l_{1}} \int_{0}^{l_{2}} B(x, y) \mathrm{d} x \mathrm{~d} y=2 \pi N
$$

where $N \geqslant 1$ is an integer. Then, the system of equations

$$
\begin{aligned}
& \chi\left(x-l_{1}, y\right)=\chi(x, y) \exp i \phi_{1}(x, y), \\
& \chi\left(x, y-l_{2}\right)=\chi(x, y) \exp i \phi_{2}(x, y),
\end{aligned}
$$

for a function $\chi(x, y)$ on $\mathbb{Z}^{2}$, is consistent. To see that, we observe that the compatibility condition for the magnetic translation operators ${ }^{6}$ in Eqs. (2.3) follows from the relation $\phi_{1}\left(x, y-l_{2}\right)$ $-\phi_{1}(x, y)+\phi_{2}(x, y)-\phi_{2}\left(x-l_{1}, y\right)=2 \pi N$. This is the value of the flux of $B$ in terms of the variations of the potential vector implied by formulas (2.2). The relevance of Eqs. (2.3) in this context is due to the following fact. We observe that a solution $\chi$ with $|\chi|=1$ serves to define a gauge transformation [Eq. (2.1)]. In such a case, we obtain a new wave function $\xi(a)$ and coefficients $v_{1}(a)$, $v_{2}(a)$ for Eq. (1.3), according to the following formulas:

$$
\begin{gathered}
u_{k}(a)=v_{k}(a) \frac{\chi(a)}{\chi\left(a-e_{k}\right)}, \\
\psi(a)=\chi(a) \xi(a) .
\end{gathered}
$$

The Schrödinger equation [Eq. (1.2)] for $\xi$ is now given in terms of the periodic coefficients $v_{k}$, $v_{k}\left(a+l_{j} e_{j}\right)=v_{k}(a)$ for $j, k=1,2$. This periodicity is a consequence of definitions (1.4) and Eqs. (2.3). In this new gauge, the vector potential is now given by $A+i \nabla \ln \chi$ in agreement with Eq. (2.1). The periodicity of the coefficients $v_{k}$ makes now possible to express the solutions of Eq. (1.3) as Bloch's wave functions,

$$
\xi(x, y)=e^{i\left(p_{1} x+p_{2} y\right)} f(x, y),
$$

where $f(x, y)$ is periodic with the periods $l_{1}, l_{2}$ of the magnetic field, $f\left(a+l_{j} e_{j}\right)=f(a)$. The resulting wave function in the original gauge is thus

$$
\psi(x, y)=e^{i\left(p_{1} x+p_{2} y\right)} \chi(x, y) f(x, y)
$$

for which we obtain a linear system of $l_{1} l_{2}$ equations in the coefficients $f(x, y)$,

$$
\begin{aligned}
& e^{-i p_{1}} v_{1}(x, y) f(x-1, y)+e^{i p_{1}} v_{1}^{-1}(x+1, y) f(x+1, y)+e^{-i p_{2}} v_{2}(x, y) f(x, y-1)+e^{i p_{2}} v_{2}^{-1}(x, y+1) f(x, y \\
& \quad+1)=E f(x, y),
\end{aligned}
$$

for $(x, y)$ in $\mathbb{Z}^{2} /\left(Z l_{1} e_{1}+Z l_{2} e_{2}\right)$ and $v_{1}, v_{2}$ defined by Eq. (2.4). The $l_{1} l_{2}$ functions $E\left(p_{1}, p_{2}\right)$ are defined on the reciprocal lattice. Then, for an integer flux, the states of an electron in $\mathbb{Z}^{2}$ in the presence of a periodic magnetic field are equivalent to Bloch functions.

\section{THE WAVE FUNCTION}

To accomplish the explicit construction of the solutions of the Schrödinger [Eq. (1.2)], we shall fix a concrete gauge to proceed to the analysis of Eqs. (2.3). We shall consider both the Coulomb and Landau gauges. The Coulomb gauge is interesting when one considers the connection of the solutions with those of the continuous problem. ${ }^{6,7}$ In the Landau gauge the final form of the solution is very simple. Each gauge is related to one of the classical descriptions of elliptic functions in the form of Weierstrass and Jacobi. ${ }^{15}$ In this section we describe the electronic states in the Coulomb gauge.

Let us consider the condition on $A, \nabla A=0$. In this Coulomb gauge the potential and the periodic magnetic field admit the representation 


$$
A_{1}=-\frac{\partial \varphi}{\partial y}, \quad A_{2}=\frac{\partial \varphi}{\partial x}, \quad B=\Delta \varphi
$$

with the function $\varphi$ given by ${ }^{6}$

$$
\varphi(x, y)=\frac{1}{2 \pi} \int_{0}^{l_{1}} \int_{0}^{l_{2}} \ln \left|\sigma\left(z-z^{\prime}\right)\right| B\left(x^{\prime}, y^{\prime}\right) \mathrm{d} x^{\prime} \mathrm{d} y^{\prime}, \quad z=x+i y .
$$

Here $\sigma$ denotes the function

$$
\sigma(z)=z \prod_{(m, n) \neq 0}\left(1-\frac{z}{z_{m n}}\right) \exp \left(\frac{z}{z_{m n}}+\frac{1}{2} \frac{z^{2}}{z_{m n}^{2}}\right), \quad z_{m n}=m l_{1}+i n l_{2} .
$$

The $\sigma$ function of Weierstrass has zeros $z_{m n}$ and satisfies the relations

$$
\begin{gathered}
\sigma\left(z+l_{1}\right)=-\sigma(z) \exp \left(2 \eta_{1} z+\eta_{1} l_{1}\right), \\
\sigma\left(z+i l_{2}\right)=-\sigma(z) \exp \left(2 \eta_{2} z+i \eta_{2} l_{2}\right),
\end{gathered}
$$

with the usual notation for the real numbers $\eta_{1}=\zeta\left(l_{1} / 2\right), \tilde{\eta}_{2}=-i \eta_{2}=-i \zeta\left(i l_{2} / 2\right)$. The $\zeta$ function is defined by $\zeta=\mathrm{d} \ln \sigma / \mathrm{d} z$ and the constants $\eta_{1}, \tilde{\eta}_{2}$ satisfy the Legendre relation $\eta_{1} l_{2}-\tilde{\eta}_{2} l_{1}=\pi$. Taking into account definition (3.1) and formulas (3.2) for the transformation of the $\sigma$ function, one easily gets the relations

$$
\begin{aligned}
& \varphi\left(x-l_{1}, y\right)=\varphi(x, y)-2 N \eta_{1} x+N \eta_{1} l_{1}+\frac{\eta_{1}}{\pi} \int_{0}^{l_{1}} \int_{0}^{l_{2}} x^{\prime} B\left(x^{\prime}, y^{\prime}\right) \mathrm{d} x^{\prime} \mathrm{d} y^{\prime}, \\
& \varphi\left(x, y-l_{2}\right)=\varphi(x, y)+2 N \tilde{\eta}_{2} y-N \tilde{\eta}_{2} l_{2}-\frac{\tilde{\eta}_{2}}{\pi} \int_{0}^{l_{1}} \int_{0}^{l_{2}} y^{\prime} B\left(x^{\prime}, y^{\prime}\right) \mathrm{d} x^{\prime} \mathrm{d} y^{\prime},
\end{aligned}
$$

when the flux of the magnetic field on its periodic parallelogram is $2 \pi N$. With the aid of these formulas we obtain the following expressions for the functions $\phi_{1}, \phi_{2}$ in Eq. (2.2):

$$
\phi_{1}(x, y)=-2 N \eta_{1} y, \quad \phi_{2}(x, y)=-2 N \tilde{\eta}_{2} x,
$$

up to an arbitrary constant, as follows from the variations of the vector potential $A$ written earlier, Eqs. (2.2). We thus arrive to the transformation laws

$$
\begin{aligned}
& \chi\left(x-l_{1}, y\right)=\chi(x, y) e^{-i 2 N \eta_{1} y}, \\
& \chi\left(x, y-l_{2}\right)=\chi(x, y) e^{-i 2 N \widetilde{\eta}_{2} x} .
\end{aligned}
$$

These equations represent conditions (2.3) for the present Coulomb gauge. To solve them, first we extend Eq. (3.3) to the entire plane $\mathbb{R}^{2}$. Now, we see that the function

$$
e^{-\beta z} e^{\varphi(x, y)} \chi(x, y)
$$

behaves under translations, in a multiple of the field periods, like the product of $N \sigma$ functions. The constant $\beta$ is determined below. Therefore, the conclusion is that we may write a solution $\chi_{0}$ of Eqs. (3.3) in the form 


$$
\chi_{0}(x, y)=e^{-\varphi(x, y)} e^{\beta z} \prod_{j=1}^{N} \sigma\left(z-\alpha_{j}\right),
$$

where the constants, $\beta$ and the sum $\varsigma=\sum_{j=1}^{N} \alpha_{j}$, are defined by the following formulas. The constant $\beta$ obtains from

$$
\begin{aligned}
& \operatorname{Re} \beta=\frac{2 \eta_{1}}{l_{1}} \operatorname{Re} s-\frac{\eta_{1}}{\pi l_{1}} \int_{0}^{l_{1}} \int_{0}^{l_{2}} x^{\prime} B\left(x^{\prime}, y^{\prime}\right) \mathrm{d} x^{\prime} \mathrm{d} y^{\prime}, \\
& \operatorname{Im} \beta=\frac{2 \tilde{\eta}_{2}}{l_{2}} \operatorname{Im} s-\frac{\tilde{\eta}_{2}}{\pi l_{2}} \int_{0}^{l_{1}} \int_{0}^{l_{2}} y^{\prime} B\left(x^{\prime}, y^{\prime}\right) \mathrm{d} x^{\prime} \mathrm{d} y^{\prime},
\end{aligned}
$$

and the quantity $s$ is defined as

$$
2 \pi i \varsigma=\pi N\left(l_{2}+i l_{1}\right)+\frac{l_{1} l_{2}}{\pi} \int_{0}^{l_{1}} \int_{0}^{l_{2}}\left(\frac{\tilde{\eta}_{2} y^{\prime}}{l_{2}}+i \frac{\eta_{1} x^{\prime}}{l_{1}}\right) B\left(x^{\prime}, y^{\prime}\right) \mathrm{d} x^{\prime} \mathrm{d} y^{\prime} .
$$

Since the zeros of $\chi_{0}$ are placed at points $\alpha_{j}$, and their translates by the field periods $l_{1}$ and $l_{2}$, the numbers $\alpha_{j}$ can be chosen in such a way that $\chi_{0}$ does not have zeros in $\mathbb{Z}^{2}$. Notice that the quantity $\varsigma$ is determined by the magnetic field that imposes a constraint on the $\alpha_{j}$, namely, $\Sigma_{j=1}^{N} \alpha_{j}=\varsigma$. If $\varsigma / N$ is not in $\mathbb{Z}^{2}$ we can take all of the $\alpha_{j}=\varsigma / N$. On the contrary, in case $\varsigma / N$ belongs to $\mathbb{Z}^{2}$ we take $\alpha_{j}=\varsigma / N+\pi \epsilon^{j} / 3$ with $\epsilon=\exp 2 \pi i / N$ since $\pi / 3$ is not an integer and the sum of the $N$ th roots of unity is equal to zero. Numbers $\left\{\alpha_{1}, \alpha_{2}, \ldots, \alpha_{N}\right\}$ merely specify an admissible gauge transformation. Gauge invariance implies that the physical state $\psi$ and the energy $E$ in the Schrödinger equation [Eq. (1.2)] do not depend on the choice made for the constants $\alpha_{j}$. The existence of the transformation reflects the difference between the problem on $\mathbb{Z}^{2}$ and the continuous problem, because in the continuous case the zeros of $\chi(x, y)$ cannot be avoided and hence this function does not define a gauge transformation from the original Schrödinger equation to a periodic equation. From our previous discussion, taking into account that $\left|\chi_{0}(x, y)\right|$ is a periodic function, we deduce that the solution $\chi$ we were looking for to Eq. (3.3) can be written in the form

$$
\chi=\frac{\chi_{0}}{\left|\chi_{0}\right|}=e^{i\left(\beta_{2} x+\beta_{1} y\right)} \prod_{j=1}^{N} \frac{\sigma\left(z-\alpha_{j}\right)}{\left|\sigma\left(z-\alpha_{j}\right)\right|} .
$$

In this formula, $\beta_{1}$ and $\beta_{2}$ are the real and imaginary parts, respectively, of $\beta=\beta_{1}+i \beta_{2}$. The final form for the solution $\psi$ of Eq. (2.5) is then

$$
\psi(x, y)=e^{i\left(q_{1} x+q_{2} y\right)} f(x, y) \prod_{j=1}^{N} \frac{\sigma\left(z-\alpha_{j}\right)}{\left|\sigma\left(z-\alpha_{j}\right)\right|},
$$

with $q_{1}=\beta_{2}+p_{1}, q_{2}=\beta_{1}+p_{2}$ and the function $f(x, y)$ is a solution of Eq. (2.6). The wave function $\psi$ can be written also as

$$
\psi(x, y)=\Psi_{0}(x, y) F(x, y),
$$

the product of a ground state of the continuous problem, 6,7

$$
\Psi_{0}(x, y)=e^{i\left(p_{1} x+p_{2} y\right)} \chi_{0}(x, y),
$$

with the periodic function $F(x, y)=f(x, y) /\left|\chi_{0}(x, y)\right|$. This follows from Eq. (2.5) and the expression given for $\chi=\chi_{0} / \chi_{0} \mid$. Thus, we conclude that the states in the lattice are given by a ground state for the continuous motion modulated by a periodic function. 


\section{THE LANDAU GAUGE}

The procedure followed in the previous section to solve the spectral problem [Eq. (1.2)] admits an equivalent formulation. We set $A_{2}=0$ and define

$$
A_{1}(x, y)=-\int_{0}^{y} B\left(x, y^{\prime}\right) \mathrm{d} y^{\prime} .
$$

In this Landau gauge, functions $\phi_{1}$ and $\phi_{2}$ in Eq. (2.3) adopt the expressions

$$
\phi_{1}=0, \quad \phi_{2}=\int_{0}^{x} \int_{0}^{l_{2}} B\left(x^{\prime}, y^{\prime}\right) \mathrm{d} x^{\prime} \mathrm{d} y^{\prime} .
$$

Thus we see that $\phi_{2}(x)$ represents the magnetic flux through the rectangle $[0, x] \times\left[0, l_{2}\right]$. Equations (2.3) for $\chi$ now are

$$
\begin{gathered}
\chi\left(x-l_{1}, y\right)=\chi(x, y), \\
\chi\left(x, y-l_{2}\right)=\chi(x, y) e^{i \phi_{2}(x)} .
\end{gathered}
$$

The periodicity of $B$ implies the relation $\phi_{2}\left(x+l_{1}\right)=\phi_{2}(x)+2 \pi N$ from which we deduce that $2 \pi N x / l_{1}-\phi_{2}(x)$ is a periodic function with period $l_{1}$. The substitution

$$
\chi(x, y)=\mu(x, y) \exp \left[i\left(\frac{2 \pi N x}{l_{1}}-\phi_{2}(x)\right) \frac{y}{l_{2}}\right]
$$

reduces Eq. (4.1) to the simpler system

$$
\begin{gathered}
\mu\left(x-l_{1}, y\right)=\mu(x, y), \\
\mu\left(x, y-l_{2}\right)=\mu(x, y) \exp \frac{2 \pi i N x}{l_{1}},
\end{gathered}
$$

for the new function $\mu$. This system can be solved in terms of the Jacobi theta function $\vartheta(z, \tau){ }^{15}$ This function is defined, for $\operatorname{Im} \tau>0$, by the series

$$
\vartheta(z, \tau)=\sum_{n \in Z} \exp \left(2 \pi i n z+\pi i n^{2} \tau\right)
$$

and satisfies the relations

$$
\begin{gathered}
\vartheta(z+1, \tau)=\vartheta(z, \tau), \\
\vartheta(z+\tau, \tau)=\vartheta(z, \tau) \exp (-\pi i \tau-2 \pi i z) .
\end{gathered}
$$

The zeros of $\vartheta(z, \tau)$ form a periodic set in the lattice $\mathbb{Z} \tau+\mathbb{Z}$ at points $(1+\tau) / 2+m+n \tau$ for integer numbers $m$ and $n$. To get a solution of Eqs. (4.2) we define the function

$$
h(z)=\vartheta\left(\frac{z}{l_{1}}, i \frac{l_{2}}{l_{1}}\right)
$$

which is a $\vartheta$ function with $\tau=i l_{2} / l_{1}$. For this function $h$ one has the relations

$$
h\left(z+l_{1}\right)=h(z),
$$




$$
h\left(z+i l_{2}\right)=h(z) \exp \left(\frac{\pi l_{2}}{l_{1}}+\frac{2 \pi y}{l_{1}}\right) \exp -\frac{2 \pi i x}{l_{1}} .
$$

Provided that the real part of $\alpha$ is zero, we can take $\mu(x, y)=h(z-\alpha) /|h(z-\alpha)|$ as our sought solution to Eqs. (4.2). The solution to Eqs. (4.1) can be written in that case

$$
\chi(x, y)=\exp \left[i\left(\frac{2 \pi N x}{l_{1}}-\phi_{2}(x)\right) \frac{y}{l_{2}}\right]\left(\frac{h(z-\alpha)}{|h(z-\alpha)|}\right)^{N} .
$$

The zeros of $h(z-\alpha)$ are located at points $\alpha+\left(l_{1}+i l_{2}\right) / 2+m l_{1}+i n l_{2}$. Thus, $\alpha$ should be chosen to avoid the presence of zeros of $h(z-\alpha)$ on $\mathbb{Z}^{2}$, for instance, $\alpha=i \pi / 3$. Periodic hopping amplitudes in this gauge are given by formulas (2.4). In view of the foregoing equations [Eq. (1.4)] we have

$$
v_{1}(x, y)=\frac{\chi(x-1, y)}{\chi(x, y)} \exp -i k(x, y), \quad v_{2}(x, y)=\frac{\chi(x, y-1)}{\chi(x, y)}
$$

where we denote

$$
k(x, y)=\int_{x-1}^{x} \int_{0}^{y} B\left(x^{\prime}, y^{\prime}\right) \mathrm{d} x^{\prime} \mathrm{d} y^{\prime}
$$

The spectrum is then determined by Eq. (2.6). Let us make a final remark about the choice of the magnetic field $B$. The Fourier series for the periodic field $B$ gives the decomposition $B=B_{0}+B_{1}$, where the term $B_{0}$ is constant and the flux of $B_{1}$ is zero. Considered as a periodic function, with periods $i_{1}$ and $i_{2}$, integer multiples of $l_{1}$ and $l_{2}$, respectively, the admissible values of $B_{0}$ are $B_{0}$ $=2 \pi N / i_{1} \dot{l}_{2}$. Since the flux of $B_{1}$ is again zero, through the new periodic parallelogram, for the present value of the constant term $B_{0}$ the number of equations in Eq. (2.6) becomes $\dot{l}_{1} \dot{l}_{2}$. Consequently, the number of eigenvalues $E\left(p_{1}, p_{2}\right)$ is also $i_{1} \dot{l}_{2}$. The examples analyzed in Ref. 2 are particular instances of our construction.

In the case of a uniform magnetic field, with rational flow $2 \pi N / M$ through each elementary cell, we can take $l_{1}=1$ and $l_{2}=M$ and chose $B$ to obtain an integer flux $B l_{1} l_{2}=2 \pi N$ as in the previous formulas. In the present situation, the problem is reduced to a one-dimensional problem, described by a Bloch function, since the gauge transformation is defined by the function $\chi(x, y)$ $=1$. This follows from the conditions $\chi(x-1, y)=\chi(x, y), \chi\left(x, y-l_{2}\right)=\chi(x, y) \exp 2 \pi N i x=\chi(x, y)$ on $\mathbb{Z}^{2}$.

${ }^{1}$ D. R. Hofstadter, Phys. Rev. B 14, 2239 (1976).

${ }^{2}$ G. Y. Oh, Phys. Rev. B 60, 1939 (1999).

${ }^{3}$ P. Fekete and G. Gumbs, J. Phys.: Condens. Matter 11, 5475 (1999).

${ }^{4}$ M. C. Chang and M. F. Yang, Phys. Rev. B 69, 115108 (2004).

${ }^{5}$ P. B. Wiegmann and A. V. Zabrodin, Phys. Rev. Lett. 72, 1890 (1994).

${ }^{6}$ B. A. Dubrovin and S. P. Novikov, Sov. Phys. JETP 52, 511 (1980).

${ }^{7}$ S. P. Novikov, Sov. Math. Dokl. 23, 298 (1981).

${ }^{8}$ A. Y. Rom, Phys. Rev. B 55, 11025 (1996).

${ }^{9}$ Y. A. Kordyukov, Commun. Math. Phys. 253, 371 (2005).

${ }^{10}$ J. Bruening, V. Geyler, and K. Pankrashkin, Commun. Math. Phys. 269, 87 (2007).

${ }^{11}$ R. Varnhagen, Nucl. Phys. B: Field Theory Stat. Syst. [FS]443, 501 (1995).

${ }^{12}$ E. H. Lieb and M. Loss, Duke Math. J. 71, 337 (1993).

${ }^{13}$ E. H. Lieb, Phys. Rev. Lett. 73, 2158 (1994).

${ }^{14}$ N. Macris and B. Nachtergaele, J. Stat. Phys. 85, 745 (1996).

${ }^{15}$ D. Husemöller, Elliptic Curves (Springer, Berlin, 1987). 